\title{
Investigation of the Surface Properties of Vinyl Ethers - Sodium 2-Acrylamido-2-Methylpropanesulfonate Copolymers
}

\author{
S. Kh. Khussain, E. M. Shaikhutdinov, N. Zh. Seitkaliyeva, A. Zh. Zhenisova \\ Department of applied chemistry, Kazakh National Technical University named after Kanysh Satpaev, Almaty, Kazakhstan \\ Email: sarah_khussain@mail.ru
}

Received 2012

\begin{abstract}
The surface properties of water soluble copolymers vinyl ethers of monoethanolamine and ethylene glycol with sodium 2-acrylamido-2-methyl-propanesulfonate were investigated by studying adsorption at the aqueous solution - air interface. It is found that copolymers have considerably higher surface activity in comparison with poly- sodium 2-acrylamido-2-methyl-propanesulfonate.
\end{abstract}

Keywords: Surface Active Copolymers; Adsorption; Interface; Standard Free Energy of Adsorption

\section{Introduction}

Investigation of the behavior of macromolecules at the interface is one of the biggest challenges because surface phenomena in polymers and polymeric materials play an important role in the whole complex of their properties, especially in their structural and mechanical properties.

The combination of the polar hydrophilic groups and nonpolar hydrophobic parts of chain in macromolecules gives to the functional areas of polymers the surface-active properties and the possibility to use them as flocculants, flotation reagents, antistatic agents, stabilizers of disperse systems, etc., used in the oil, pharmaceutical and textile industry, metallurgy, agriculture and for other purposes.

We have synthesized new multifunctional copolymers of vinyl ethers of monoethanolamine and ethylene glycol with sodium 2-acrylamido-2-methyl-propanesulfonate by free- radical copolymerization in aqueous medium $[1,2]$.

This report is devoted to the determination of surface-active properties of these copolymers by studying the adsorption at the aqueous solution-air interface.

\section{Experimental}

The Na-AMPS monomer was prepared from 2-acrylamido-2methylpropanesulfonic acid (H-AMPS) (the content of the main product was no less than 99 wt \%) purchased from Avocado Research Chemicals Ltd. (Switzerland).

Ethylene glycol vinyl ether (EGVE) and monoethanolamine vinyl ether (MEAVE) were purified by vacuum distillation $\left(T_{\mathrm{b}}=72 \square \mathrm{C} / 30 \mathrm{kPa}, n_{D}{ }^{20}=1.4356\right.$ and $T_{\mathrm{b}}=45-46 \square \mathrm{C} / 1.333 \mathrm{kPa}$, $n_{D}{ }^{20}=1.4357$ respectively).

The free-radical copolymerization of Na-AMS and MEAVE was performed in an inert medium at $60^{\circ} \mathrm{C}$ at various molar ratios of the starting monomers in aqueous solution. The reaction was carried out in the presence of an equimolar mixture of sodium bisulfite and potassium persulfate as a redox initiator. The weight of the initiator was $0.1 \%$ with respect to the total weight of comonomers.

The composition of the copolymer was determined by IRspectroscopy and potentiometric titration on an EV-74 ionometer with the use of glass and silver chloride electrodes.

Adsorption of copolymers at the air-water solution interface was studied by measuring surface tension. Surface tension $(\sigma)$ of polymer sodium 2-acrylamido-2-methyl-propanesulfonate (Na-AMPS) aqueous solution, copolymer of ethylene glycol vinyl ether- sodium 2-acrylamido-2-methyl- propanesulfonate (EGVE-Na-AMPS), and copolymer of monoethanolamine vinyl ether - sodium 2-acrylamido-2-methyl- propanesulfonate (MEAVE-Na-AMPS) was measured at $298 \mathrm{~K}$ by the Wilhelmy [3]. The $\sigma$ value of solutions was calculated according to the equation [4]:

$$
\sigma=\frac{\left({ }^{m} p \bar{a}^{m}\right) g}{2(l+d)}
$$

where $m_{p}$ and $m_{a}$ - the mass of the plate in the solution and air, respectively; $g$ - gravitational acceleration; $l$ and $d$ - the width and thickness of the submerged part of the plate, respectively.

Retraction force of the plate to the solution was measured using a torsion balance to an accuracy of $\pm 10^{-6} \mathrm{~kg}$.

To determine the equilibrium value of surface tension, the measurement for each solution thermostatically-controlled to an accuracy of $\pm 0.5^{0} \mathrm{C}$ was performed after 24 hours. The average value $\sigma$ was found then from several measurements. The accuracy of surface tension measurement did not exceed $\pm 0,3 \mathrm{mN}$ / $\mathrm{m}$.

\section{Results and Discussion}

Figures 1 and 2 show kinetics of surface tension reduction of the copolymer aqueous solution depending on adsorption time. 
It is evident that, for aqueous solutions of the copolymer, the equilibrium value of surface tension $(\sigma)$ is reached during several hours, which is typical for high molecular surface active compounds.

The adsorption of macromolecules at interface has its own peculiarities, among which the most important - the slowness of the process [5]. It manifests itself, particularly, in reducing the surface tension of aqueous solutions of macromolecular substances in the range of long time. The process can be divided in two stages:

1) the diffusion of macromolecules at the interface

2) the restructuring of certain segments of the macromolecules on polarity at the interface under the action of the surface force field.

In order to obtain information on the duration of the copolymer adsorption the relaxation times of adsorbed layers were calculated on kinetic date according to equation [6]:

$$
\lg \left(\sigma_{\tau}-\sigma_{\infty}\right)=\lg \left(\sigma_{0}-\sigma_{\infty}\right)-\tau / 2,3 \vartheta,
$$

where $\sigma_{\tau}$ - surface tension value of solution at time $\tau, \mathrm{mN} / \mathrm{m}$; $\sigma_{0}$ - the initial of surface tension at $\tau=0, \mathrm{mN} / \mathrm{m} ; \sigma_{\infty}$ - the equilibrium value of surface tension (after $24 \mathrm{~h}$.), $\mathrm{mN} / \mathrm{m} ; \vartheta$ relaxation time of the adsorption layer, min.

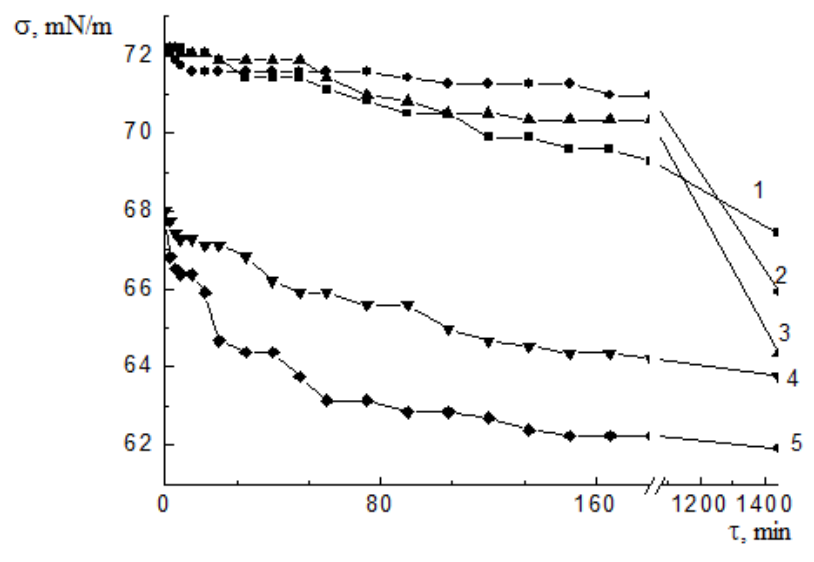

$\mathrm{m}_{1}=24.7 \mathrm{~mol}$. MEAVE \% in the polymer composition

Figure 1. Kinetics of the surface tension reduction of polymer MEAVE - Na-AMPS at different concentrations of aqueous solutions (wt \%) in solution: 0.02 (1), 0.04 (2), 0.09 (3), 0.12 (4), 0.5 (5).

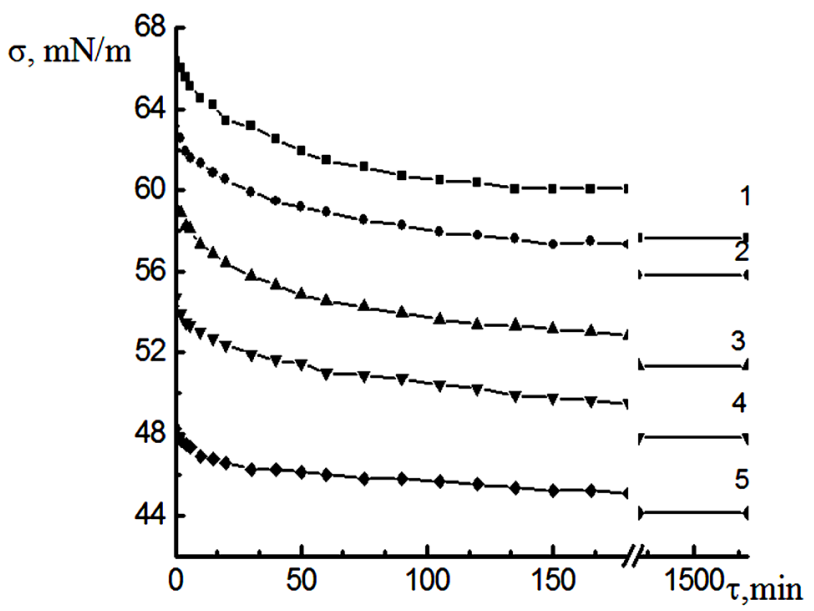

$\mathrm{m}_{1}=30$ mol.\% VEEG in the polymer composition

Figure 2. Kinetics of the surface tension reduction of polymer VEEG - Na-AMPS at different concentrations of aqueous solutions (wt \%): 0.02 (1), 0.04 (2), 0.06 (3), 0.08 (4), 0.12 (5)

The relaxation times of polymer adsorption layers VEMEA Na-AMPS and VEEG-Na-AMPS at the interface solution - air are shown in Table $\mathbf{1}$.

Analysis of the data (Table 1) shows that in the case of the copolymer VEMEA-Na-AMPS relaxation time is directly proportional to the concentration of the copolymer, whereas for the copolymer VEEG-Na-AMPS, this dependence passes through a maximum. Obviously, this is due to the conformation of the molecules of copolymers. At low concentrations of the solution of the copolymer macromolecules are in a more expanded conformation and reorientation of the branched structure of the copolymer VEEG-Na-AMPS takes more time. Subsequent increase of copolymer solution concentration raises quantity of simultaneously adsorbing macromolecules which leads to decrease of vacant place on surface. As a result the reorientation of macromolecular segments on polarity at the air- solution interface becomes difficult and, hence, relaxation time of adsorption layer decrease [6].

The isotherm of surface tension of copolymer solution VEEG - Na-AMPS and MEAVE - Na-AMPS based on equilibrium value of $\sigma$ was constructed (Figure 3, curve 2,3), together with

Table 1. The relaxation time of adsorption layers of copolymers MEAVE - Na-AMPS and VEEG - Na-AMPS at different concentrations of solution.

\begin{tabular}{ccc}
\hline Copolymer & Concentration of copolymer, wt. \% & Relaxation time 9, min. \\
\hline & 0,02 & 145 \\
MEAVE - & 0,04 & 147 \\
Na-AMPS & 0,09 & 150 \\
& 0,12 & 152 \\
& 0,50 & 172 \\
& 0,02 & 190 \\
VEEG-Na-AMPS & 0,04 & 423 \\
& 0,06 & 500 \\
& 0,08 & 126 \\
\hline
\end{tabular}




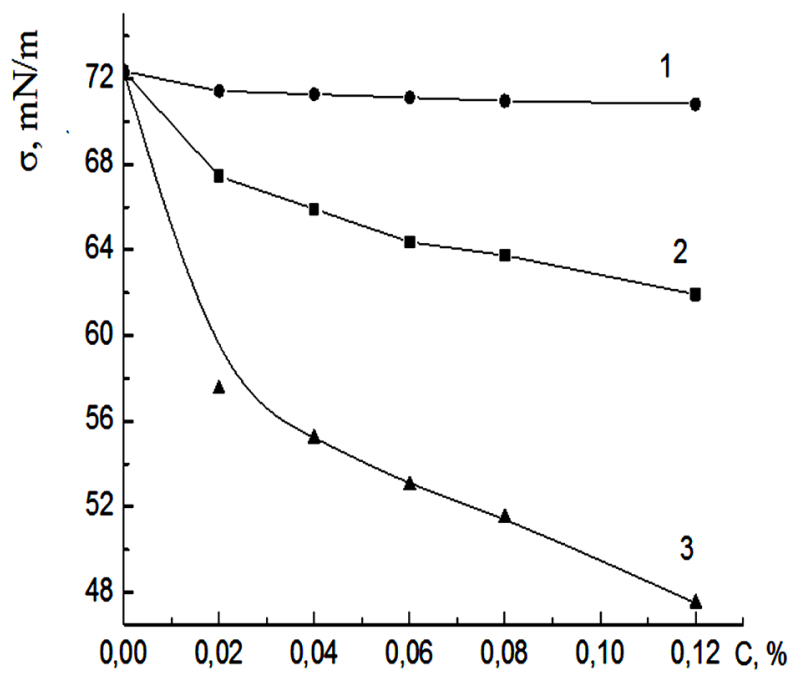

Figure 3. Isotherms of surface tension of aqueous solutions polyNaAMPC (1) and copolymer MEAVE - Na-AMPS (3), copolymer VEEG - Na-AMPS (2).

the isotherm of surface tension water solution poly- Na-AMPS

(Figure 3, curve 1).

As can be seen from the Figure 3, the curve $\sigma=f(c)$ of co- polymer MEAVE - Na-AMPS is below the curve VEEG - NaAMPS, which indicates that surface activity of monoethanolamine vinyl ether copolymer is higher compared to VEEG Na-AMPS. The higher surface activity polymer VEMEA-NaAMPS explained by the formation of intramolecular salt bond between amino- and sulfonic acid groups, resulting in increased hydrophobicity of the copolymer and is its compactness:

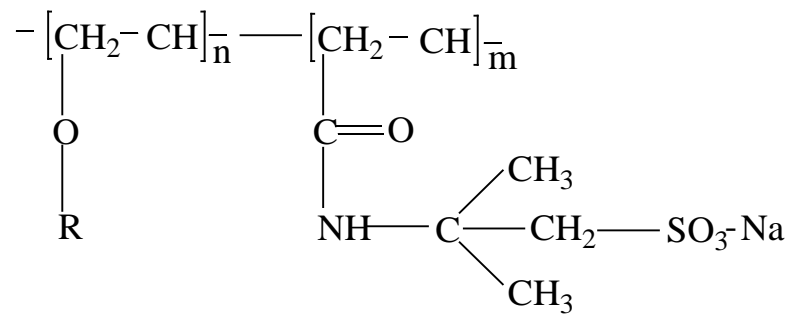

where $\mathrm{R}-\left(\mathrm{CH}_{2}\right)_{2}-\mathrm{OH}$, and $-\left(\mathrm{CH}_{2}\right)_{2}-\mathrm{NH}_{2}$

Based on isotherms the surface activity on Rebinder $\left(\mathrm{G}_{\mathrm{Re}}\right)$ for poly- Na-AMPS and copolymers was determined according to equation [7] (Table 2):

$$
G_{\mathrm{Re}}=\lim \left(-\frac{d \sigma}{d c}\right)_{c \rightarrow 0}
$$

Table 2 demonstrates that surface activity of copolymer exceeds ones of homopolymer approximately more than 3 times.

Table 2. Physical - chemical properties of surface layers of polymers.

\begin{tabular}{lcc}
\hline \multicolumn{1}{c}{ The system } & $\mathrm{G}_{\mathrm{Re}} \cdot 10^{-3},\left(\mathrm{mN} \mathrm{m}^{-1}\right) /\left(\mathrm{kmole} \mathrm{m}^{-3}\right)$ & $\Delta_{\text {ads }} \mathrm{G}^{0}{ }_{298}, \mathrm{~kJ} /$ mole \\
\hline Poly-Na-AMPS & 1,5 & 18,0 \\
Copolymer MEAVE - Na-AMPS & 9,1 & 22,6 \\
Copolymer VEEG - Na-AMPS & 5,2 & 21,2 \\
\hline
\end{tabular}

The values of polymer's standard free energy of adsorption ( $\Delta_{\text {ads }} G^{0}{ }_{298}$ ) were calculated in order to identify the causes and mechanism of change in surface activity and adsorption. In addition, it is important characteristic of a spontaneous accumulation of substance at the interface and is a measure of the surface active macromolecule's desire to adsorb.

Standard free energy of adsorption was calculated according to the equation [8]:

$$
\Delta_{\text {ads }} \mathrm{G}_{298}^{0}=-\mathrm{RT} \ln \mathrm{G}_{\mathrm{Re}},
$$

where $\mathrm{T}$ - the absolute temperature, $\mathrm{R}$ - the universal gas constant.

As seen from the values shown in Table 2, the gain in standard free energy adsorption in transition from homopolymer to copolymer VEEG- Na-AMPS is about $3 \mathrm{~kJ} /$ base-mole, and to copolymer MEAVE - Na-AMPS is 4,6 kJ/base-mole.

Thus, the results of this study lead us to conclude that in aqueous solutions the MEAVE - Na-AMPS and VEEG-NaAMPS copolymer have higher surface activity and adsorption at the interface solution-air proceeds easier than poly-NaAMPS, i.e. vinyl ether units in the polymer chain increases the surface activity of macromolecules.

\section{REFERENCES}

[1] K. Zh. Abdiyev, S. Kh. Khussain, N. Zh. Seitkaliyeva, "New macromolecular surface active substant based on monoethanolamine vinyl ether,” Vestn. Kazakh. Nats. Univ., No.2, vol. 31, pp. 321-323, 2003.

[2] E. M. Shaikhutdinov, S. Kh. Khussain, K. Zh. Abdiyev, A. Zh. Zhenisova, N. Zh. Seitkaliyeva, "New copolymers of 2-acrylamido-2-methyl-propanesulfonic acid, and vinyl ethers," Proc. XVIII Mendeleev Congress on General and Applied Chemistry. -Moscow, vol. 2, p. 608, 2007.

[3] V. A. Kabanov, I. M. Papisov, "Complex formation between complementary synthetic polymers and oligomers in dilute solutions," Visokomolec. soiyed., No. 2, vol. 21A, pp. 243 - 261, 1979.

[4] A. Adamson, "Physical Chemistry of Surfaces," Moscow, 1979, pp. 109 - 118.

[5] D. J. Adams, M. T. A. Evans, J. R. Mitchell, M. C. Phillips, P. M. Rees, "Adsorption of Lysozime and some Acetyl derivatives at the Air-Water Interface,” J. Polym. Sci., Part C, No 34, pp. 167 169, 1971

[6] A. A. Trapeznikov, V. G. Vince, T. Yu. Shirokova, "The kinetics of the reduction of surface tension in solutions of proteins,” Colloid. Zh., No 2, vol. 43, pp. 322 - 329, 1981. 
[7] K. F. Zhigach, P. A. Rebinder, "Surface activity of hydrophilic colloid“, Zh. Phyz. Khim., vol. 13, pp. 94 - 105, 1939.

[8] V. G. Babak, M. A. Anchipolovsky, G. A. Vikhoreva, I. G. Lukina, "The mechanism of the synergistic action of bromide and tetradetciltrimetilammonium and karboximetilhitin forming surface active substance-polyelectrolyte complexes on the surface tension of mixed aqueous solutions," Colloid. Zh., No 2, vol. 58, pp. 155-162, 1996. 